\title{
The significance of PIWI family expression in human lung embryogenesis and non-small cell lung cancer
}

\author{
Alfons Navarro ${ }^{1}$, Rut Tejero ${ }^{1}$, Nuria Viñolas ${ }^{2}$, Anna Cordeiro ${ }^{1}$, Ramon M. Marrades ${ }^{3}$, \\ Dolors Fuster $^{1}$, Oriol Caritg ${ }^{1}$, Jorge Moises ${ }^{3}$, Carmen Muñoz ${ }^{1}$, Laureano Molins ${ }^{5}$, \\ Josep Ramirez ${ }^{4}$, Mariano Monzo ${ }^{1}$ \\ ${ }^{1}$ Molecular Oncology and Embryology Laboratory, Human Anatomy Unit, School of Medicine, University of Barcelona, \\ IDIBAPS, Barcelona, Spain \\ ${ }^{2}$ Department of Medical Oncology, Institut Clinic Malalties Hemato-Oncològiques (ICMHO), Hospital Clinic de Barcelona, \\ University of Barcelona, IDIBAPS, Barcelona, Spain \\ ${ }^{3}$ Department of Pneumology, Institut Clínic del Tórax (ICT), Hospital Clinic de Barcelona, University of Barcelona, IDIBAPS, \\ CIBER de Enfermedades Respiratorias (CIBERES), Barcelona, Spain \\ ${ }^{4}$ Department of Pathology, Centro de Diagnóstico Biomédico (CDB), Hospital Clinic de Barcelona, University of Barcelona, \\ IDIBAPS, CIBERES, Barcelona, Spain \\ ${ }^{5}$ Department of Thoracic Surgery, Institut Clínic del Tórax (ICT), Hospital Clinic de Barcelona, University of Barcelona, \\ Barcelona, Spain
}

Correspondence to:

Alfons Navarro, e-mail: anavarroponz@ub.edu

Keywords: PIWI proteins, piwiRNAs, PIWIL1, PIWIL4, NSCLC

Received: November 14,2014 Accepted: December 21, $2014 \quad$ Published: January 23, 2015

\section{ABSTRACT}

The expression of Piwi-interacting RNAs, small RNAs that bind to PIWI proteins, was until recently believed to be limited to germinal stem cells. We have studied the expression of PIWI genes during human lung embryogenesis and in paired tumor and normal tissue prospectively collected from 71 resected non-small-cell lung cancer patients. The mRNA expression analysis showed that PIWIL1 was highly expressed in 7-week embryos and downregulated during the subsequent weeks of development. PIWIL1 was expressed in 11 of the tumor samples but in none of the normal tissue samples. These results were validated by immunohistochemistry, showing faint cytoplasmic reactivity in the PIWIL1-positive samples. Interestingly, the patients expressing PIWIL1 had a shorter time to relapse (TTR) $(p=0.006)$ and overall survival (OS) $(p=0.0076)$ than those without PIWIL1 expression. PIWIL2 and 4 were downregulated in tumor tissue in comparison to the normal tissue $(p<0.001)$ and the patients with lower levels of PIWIL4 had shorter TTR $(p=0.048)$ and OS $(p=0.033)$. In the multivariate analysis, PIWIL1 expression emerged as an independent prognostic marker. Using 5-Aza-dC treatment and bisulfite sequencing, we observed that PIWIL1 expression could be regulated in part by methylation. Finally, an in silico study identified a stem-cell expression signature associated with PIWIL1 expression.

\section{INTRODUCTION}

Non-small-cell lung cancer (NSCLC) accounts for $85 \%$ of all lung cancers and has a 5-year survival rate of $16 \%$ [1]. Surgery is the initial treatment for earlystage NSCLC patients, but even after complete resection, recurrence rates are substantial (20-85\%, depending on tumor stage) [2], highlighting the need for prognostic markers. From a stem-cell point of view, a tumor can be viewed as an aberrant organ initiated by a tumorigenic cancer cell that acquires the capacity for indefinite proliferation through accumulated mutations [3], indicating that the study of embryogenesis can be a good source of prognostic markers. Several studies have shown that cancer recapitulates the gene expression pattern found in the early development of the corresponding organ [4-7] and genes that are functionally altered in lung cancer, including noncoding RNA genes, play a key role in lung development [8]. 
The recognition of the importance of non-coding RNAs in cancer biology has increased considerably in recent years, with a special focus on small non-coding RNAs ( $<200$ bp long) [9]. One of the most frequent functions of small non-coding RNAs is the silencing of RNA expression by a base pairing interaction mechanism [10], which always involves a member of the Argonaute family that binds to the small non-coding RNAs. The PIWI protein subfamily of Argonaute proteins is composed of four members in humans: PIWIL1 (HIWI), PIWIL2 (HILI), PIWIL3 and PIWIL4 (HIWI2) [11]. The PIWI proteins bind to Piwi-interacting RNAs (piRNAs) [12], which are small, 24-32nt long, single-stranded RNAs. Until recently, piRNAs were believed to be expressed exclusively in the germ line $[13,14]$, but they are now known to be more universally expressed and have been detected in somatic and tumor cells [15-17]. Multiple functions have been associated with the PIWI/piRNA pathway, including the repression of transposons, which can multiply and move to new positions in the genome. By repressing transposons, the PIWI/piRNA pathway thus procures the genomic stability of germline cells [18]. piRNAs also target mRNAs, leading to their degradation, and are involved in epigenetic regulation in the nucleus of the cell $[19,20]$.

piRNA biogenesis is Dicer-independent and mediated by PIWI proteins [21]. Two main pathways of piRNA biogenesis have been described: the primary pathway and the secondary pathway (also known as the "ping-pong" amplification cycle). Little is known about the molecules that participate in piRNA biogenesis in humans, but PIWIL1 is known to act exclusively in the primary pathway and PIWIL4 in the secondary pathway, while PIWIL2 acts in both [14]. The primary pathway is believed to produce new piRNAs, while the secondary pathway is believed to be in charge of maintaining the total pool of active piRNAs in the cell [22-24].

In several cancer cell lines, PIWIL1 overexpression has been related to cell proliferation [25-27] and PIWIL2 overexpression to anti-apoptotic signaling and cell proliferation $[28,29]$. Interestingly, PIWI genes are associated with stem cell self-renewal and are re-expressed in precancerous stem cells that have the potential for malignant differentiation [30, 31]. The first report of PIWI expression in tumor tissue was in seminomas, where PIWIL1 was detected in the tumor but not in normal tissue [22]. PIWIL1 overexpression has since been detected in sarcomas, where it drives tumorogenesis via increased global DNA methylation [32], in colorectal cancer [33], where it plays a role as a prognostic marker, and in other tumors (reviewed in [34]). However, the role of PIWI genes in NSCLC has not been studied.

We hypothesized that the PIWI/piRNA pathway may be an embryonic mechanism that is inactivated in adult differentiated lung cells and reactivated during tumorogenesis. To clarify this role of PIWI genes, we have examined their expression in human embryonic lungs
(Figure 1A and 1B) and in paired tumor and normal tissue from surgically resected NSCLC patients and correlated our findings with patient outcome.

\section{RESULTS}

\section{PIWI family expression during human lung embryogenesis}

The expression of the PIWI genes PIWIL1-4 was assessed in human embryonic lung tissue from embryos of 6-13 weeks of development (Figure 1C-1F). The median $2^{-\Delta \mathrm{Ct}}$ values were 0.0084 and 0.0055 for PIWIL4 and PIWIL2, respectively, in comparison with 0.00014 and 0.000057 for PIWIL1 and PIWIL3, respectively. PIWIL4 thus had the highest expression levels. When we compared embryonic PIWI expression longitudinally from 6 to 13 weeks, significant changes in expression were observed only in PIWIL1 and PIWIL2 levels as the lung became more differentiated. PIWIL1 showed a significant reduction in overall levels from week 6 to 13 (Figure 1C), while PIWIL2 showed a curved expression with an increase beginning at week 8 , peaking at week 10 , and decreasing to initial levels at week 13 (Figure 1D).

\section{PIWI family expression in tumor and normal tissue samples and correlation with clinical characteristics}

Seventy-one patients were included in the analysis. All patients had pathologically confirmed stage I-III NSCLC and Eastern Cooperative Oncology Group performance status $0-1$ (Table 1). In tumor tissue, PIWIL1 expression was detected in eleven patients (15.5\%), PIWIL2 in $66(93 \%)$, PIWIL3 in five $(7 \%)$ and PIWIL4 in 60 (84.5\%). In normal tissue, only PIWIL2 and PIWIL4 were expressed, while PIWIL1 and PIWIL 3 were not detected in any of the 71 normal tissues examined. Both PIWIL2 (Figure 2A) and PIWIL4 (Figure 2B) were significantly downregulated in tumor tissue compared to normal tissue $(p<0.001)$.

PIWIL2 and PIWIL4 were downregulated in current and former smokers compared to never smokers ( $p=0.01$ and $p=0.003$, respectively). PIWIL2 was also downregulated in patients with lymph node involvement (N0 vs N1/2; $p=0.038$ ). No other correlation with clinical or molecular characteristics and PIWIL1-4 expression was observed.

\section{PIWIL1 and PIWIL4 expression and clinical outcome}

The 11 patients expressing PIWIL1 (PIWIL1 Ct < 35 and $\beta$-actin $<35$ ) had shorter TTR (22 vs 55 months; $p=0.006)$ and OS (32 vs 61 months; $p=0.0076)$ than those not expressing PIWIL1 (Figure 3A-3B). Using the cut-off 
A

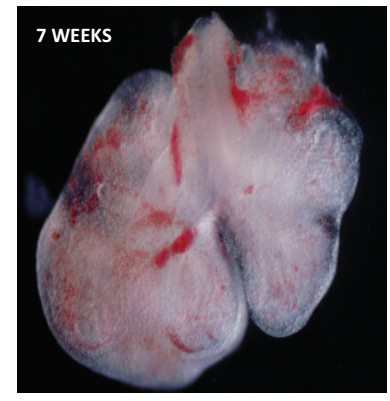

C

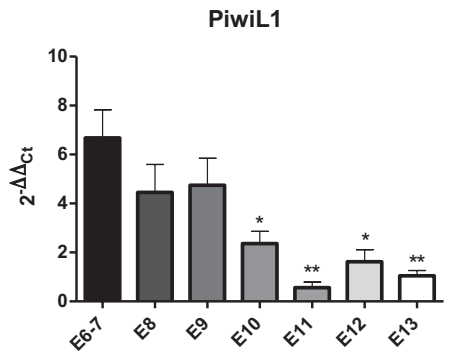

$\mathbf{E}$

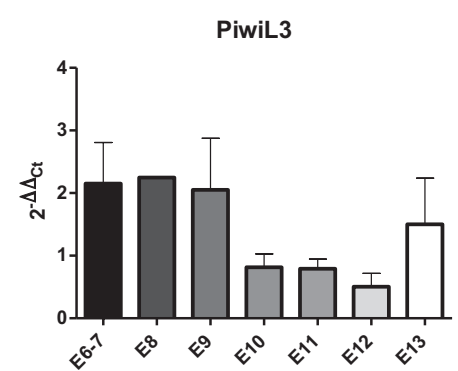

B

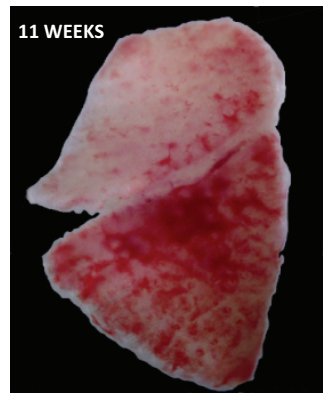

D

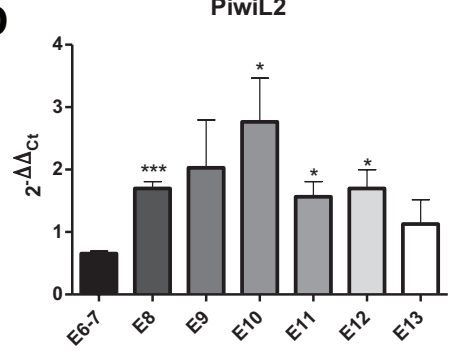

$\mathbf{F}$

PiwiL4

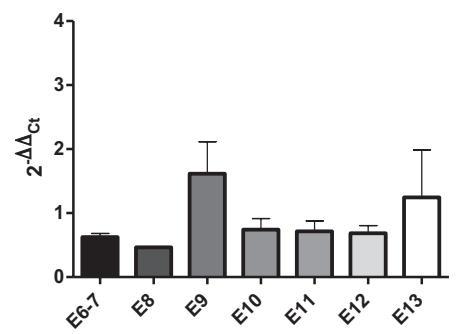

Figure 1: Images of the embryonic lung at (A) 7 weeks and (B) 13 weeks. Expression levels of (C) PIWIL1, (D) PIWIL2, (E) PIWIL3, and (F) PIWIL4 in the lung from week 6 to 13 of embryonic development.

Table 1: Patient characteristics

\begin{tabular}{|c|c|c|c|c|}
\hline \multirow[t]{2}{*}{ Characteristics } & \multirow[t]{2}{*}{ Value } & \multirow[t]{2}{*}{$N=71 \mathrm{~N}(\%)$} & \multicolumn{2}{|c|}{$p$-value* } \\
\hline & & & TTR & OS \\
\hline \multirow[t]{2}{*}{ Sex } & Male & $60(84.5)$ & & \\
\hline & Female & $11(15.5)$ & 0.099 & 0.059 \\
\hline \multirow[t]{3}{*}{ Age, yrs. } & Median (Range) & $68(46-83)$ & & \\
\hline & $<=65$ & $31(43.7)$ & & \\
\hline & $>65$ & $40(56.3)$ & 0.183 & 0.122 \\
\hline \multirow[t]{2}{*}{ ECOG PS } & 0 & $7(9.9)$ & & \\
\hline & 1 & $64(90.1)$ & 0.971 & 0.231 \\
\hline \multirow[t]{3}{*}{ Stage } & I & $44(62)$ & & \\
\hline & II & $17(23.9)$ & & \\
\hline & III & $10(14.1)$ & 0.055 & 0.008 \\
\hline
\end{tabular}




\begin{tabular}{|c|c|c|c|c|}
\hline \multirow[t]{2}{*}{ Characteristics } & \multirow[t]{2}{*}{ Value } & \multirow[t]{2}{*}{$N=71 \mathrm{~N}(\%)$} & \multicolumn{2}{|c|}{$p$-value* } \\
\hline & & & TTR & OS \\
\hline \multirow[t]{2}{*}{ Histology } & Adenocarcinoma & $40(56.3)$ & & \\
\hline & Squamous cell carcinoma & $31(43.7)$ & 0.845 & 0.313 \\
\hline \multirow[t]{3}{*}{ Type of surgery } & Lobectomy/Bilobectomy & $60(84.5)$ & & \\
\hline & Pneumonectomy & $4(5.6)$ & & \\
\hline & Atypical resection & $7(9.9)$ & 0.830 & 0.491 \\
\hline \multirow[t]{4}{*}{ Smoking history } & Current smoker & $24(33.8)$ & & \\
\hline & Former smoker & $41(57.7)$ & & \\
\hline & Never smoker & $4(5.6)$ & & \\
\hline & Unknown & $2(2.8)$ & 0.315 & 0.109 \\
\hline \multirow[t]{2}{*}{ Adjuvant treatment } & Yes & $19(26.8)$ & & \\
\hline & No & $52(73.2)$ & 0.738 & 0.740 \\
\hline \multirow[t]{2}{*}{ Relapse } & No & $45(63.4)$ & & \\
\hline & Yes & $26(36.6)$ & & \\
\hline \multirow[t]{3}{*}{ p53 mutations } & Yes & $13(18.3)$ & & \\
\hline & No & $55(77.5)$ & & \\
\hline & Unknown & $3(4.2)$ & 0.181 & 0.905 \\
\hline \multirow[t]{3}{*}{ K-ras mutations } & Yes & $10(14.1)$ & & \\
\hline & No & $56(78.9)$ & & \\
\hline & Unknown & $5(7)$ & 0.533 & 0.391 \\
\hline
\end{tabular}

*log-rank $p$-value for comparison of groups in univariate analysis.

TTR, time to relapse; OS, overall survival.

A

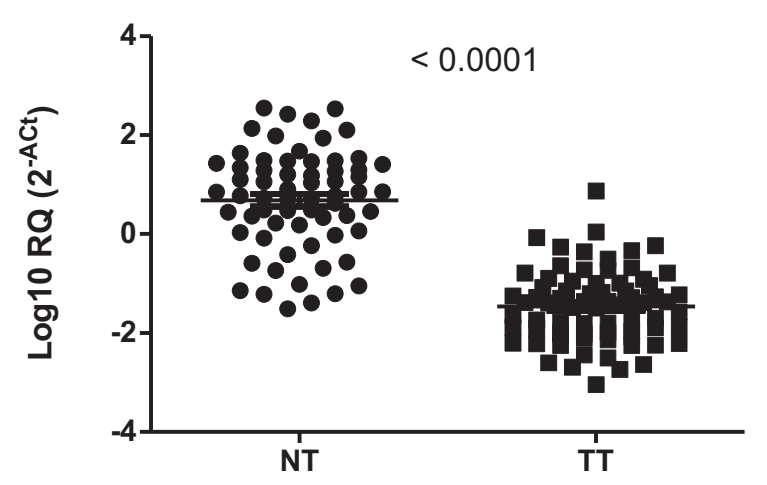

B

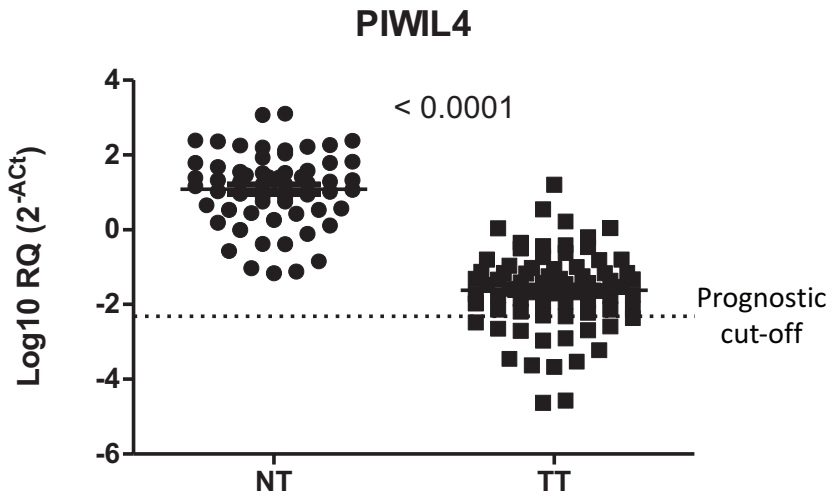

Figure 2: Expression of (A) PIWIL2 and (B) PIWIL4 in paired normal (NT) and tumor tissue (TT) from patients with non-small-cell lung cancer.

identified by the Maxstat package of $\mathrm{R}$ [35], we observed that patients with lower levels of PIWIL4 (PIWIL4 log10 $\left[2^{-\Delta \mathrm{Ct}}\right] \leq-2.31$, percentile $21.5 \%$, Figure 2 ) had shorter TTR (30 vs 56 months; $p=0.048$ ) and OS (36 vs 62 months; $p=0.033$ ) than those with high levels (Figure 3C-3D).
In the multivariate analyses, PIWIL1 expression emerged as an independent prognostic factor for TTR (OR, 2.892, 95\% CI: 1.132-7.388; $p=0.026)$ and OS (OR, 2.833, 95\% CI: 1.044-7.685; $p=0.041)$ (Table 2). 
Table 2: Multivariate analyses

Time to Relapse
\begin{tabular}{|l|c|c|}
\hline Male sex & - & Odds Ratio (95\% CI) \\
\hline Stage I & - & 0.119 \\
\hline PIWIL1 expression & $2.892(1.132-7.388)$ & 0.252 \\
\hline Low PIWIL4 expression levels & - & 0.026 \\
\hline Overall Survival & Odds Ratio (95\% CI) & 0.089 \\
\hline Male sex & $6.668(1.384-32.116)$ & $p$-value \\
\hline Stage I & $0.270(0.091-0.802)$ & 0.018 \\
\hline PIWIL1 expression & $2.833(1.044-7.685)$ & 0.018 \\
\hline Low PIWIL4 expression levels & & 0.041 \\
\hline
\end{tabular}
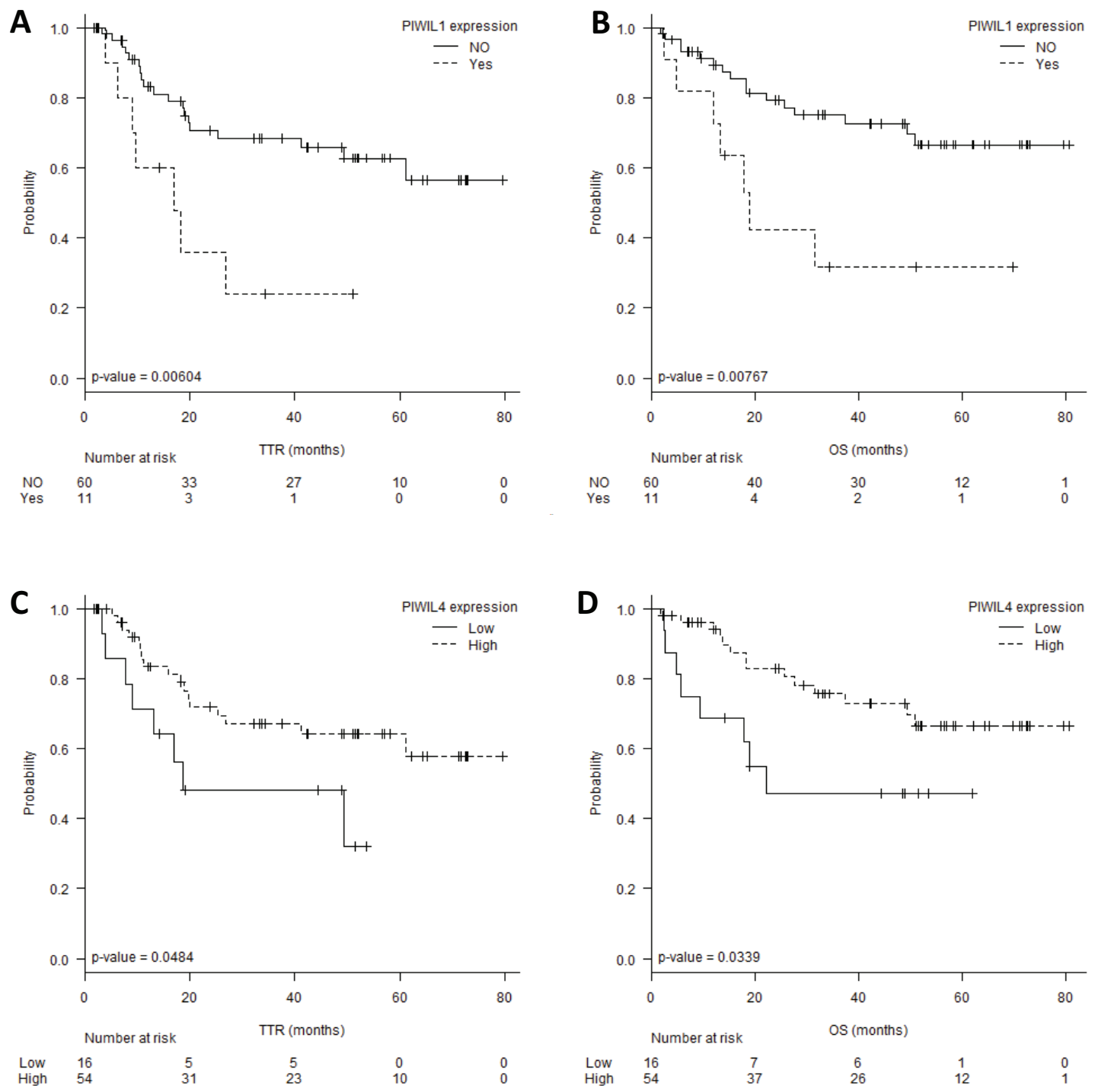

Figure 3: (A) Time to relapse (TTR) and (B) overall survival (OS) according to PIWIL1 expression levels. (C) TTR and (D) OS according to PIWIL4 expression levels. 


\section{PIWIL1 analysis by immunohistochemistry}

The study of PIWIL1 expression was validated at protein level by immunohistochemistry in 20 NSCLC cases (10 PIWIL1-positive and 10 PIWIL1-negative). The PIWIL1-positive cases showed faint cytoplasmic reactivity (Figure 4). Interestingly, the study by immunohistochemistry of embryonic samples showed that the expression of PIWIL1 was mainly found in the epithelial compartment of the embryonic lung (Figure 4A and 4B). Figure $4 \mathrm{C}$ and $4 \mathrm{D}$ show PIWIL1 expression in tumor tissue from a patient with squamous cell lung cancer. Figure 4E and $4 \mathrm{~F}$ show PIWIL1 expression in a tumor tissue from a patient with lung adenocarcinoma.
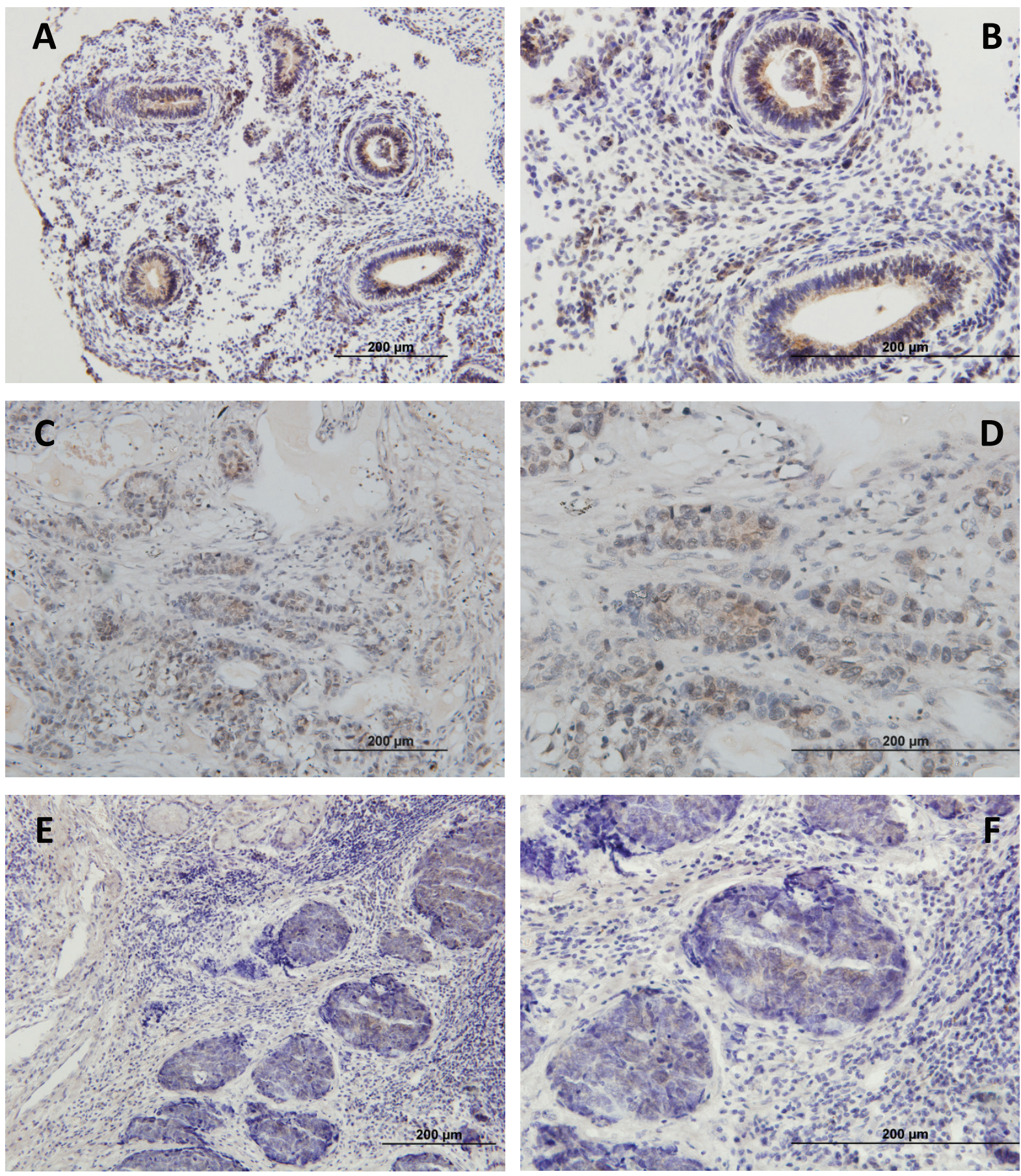

Figure 4: Immunohistochemical analysis of PIWIL1 expression in (A, B) embryonic lung tissue, (C, D) tumor tissue from a patient with squamous cell lung cancer, $(\mathbf{E}, \mathbf{F})$ tumor tissue from a patient with lung adenocarcinoma. The images in the right column are a magnification of the images in the left column. 


\section{$P I W I$ family gene expression is partially regulated by methylation and correlates with overall methylation levels}

Since PIWI genes had previously been described as regulated by methylation in human seminomas [36], we hypothesized that they could also be regulated by methylation in NSCLC. We treated two NSCLC cell lines A549 and $\mathrm{H} 23$ with the demethylating agent 5-Aza$\mathrm{dC}$ and analyzed the expression of the four PIWI genes. A dose-dependent increase in the expression levels of the four genes after 5-Aza-dC treatment was observed
(Figure 5A). We then focused further analyses on PIWIL1. We performed bisulfite sequencing analysis of the $43 \mathrm{CpG}$ island located in its promoter region in 4 samples (one 8-week embryonic lung, one 13-week embryonic lung, one PIWIL1-positive tumor and one PIWIL1-negative tumor) (Figure 5B). The 8-week embryonic lung showed the highest unmethylation level (20.5\%). The PIWIL1positive case showed higher unmethylation (17.2\%) than the PIWIL1-negative case (6.1\%) (Figure 5B).

Finally, since epigenetic functions have been associated with piRNAs, we examined the relation between the expression of the PIWI genes and overall

A

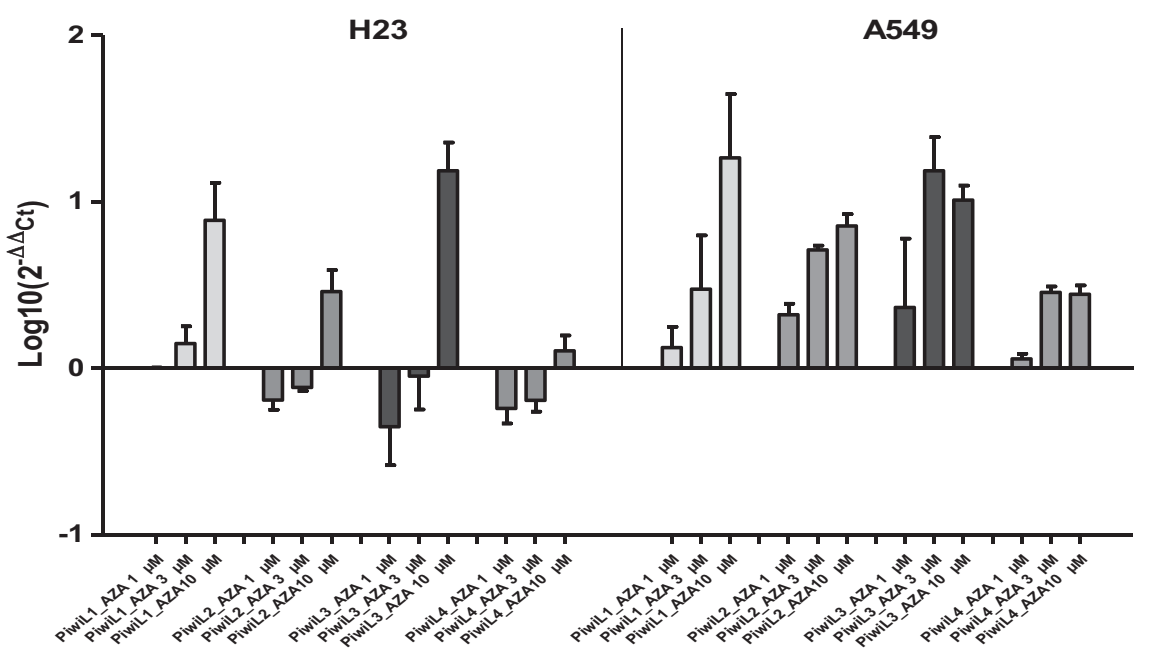

B

Chr12 (q24.33)

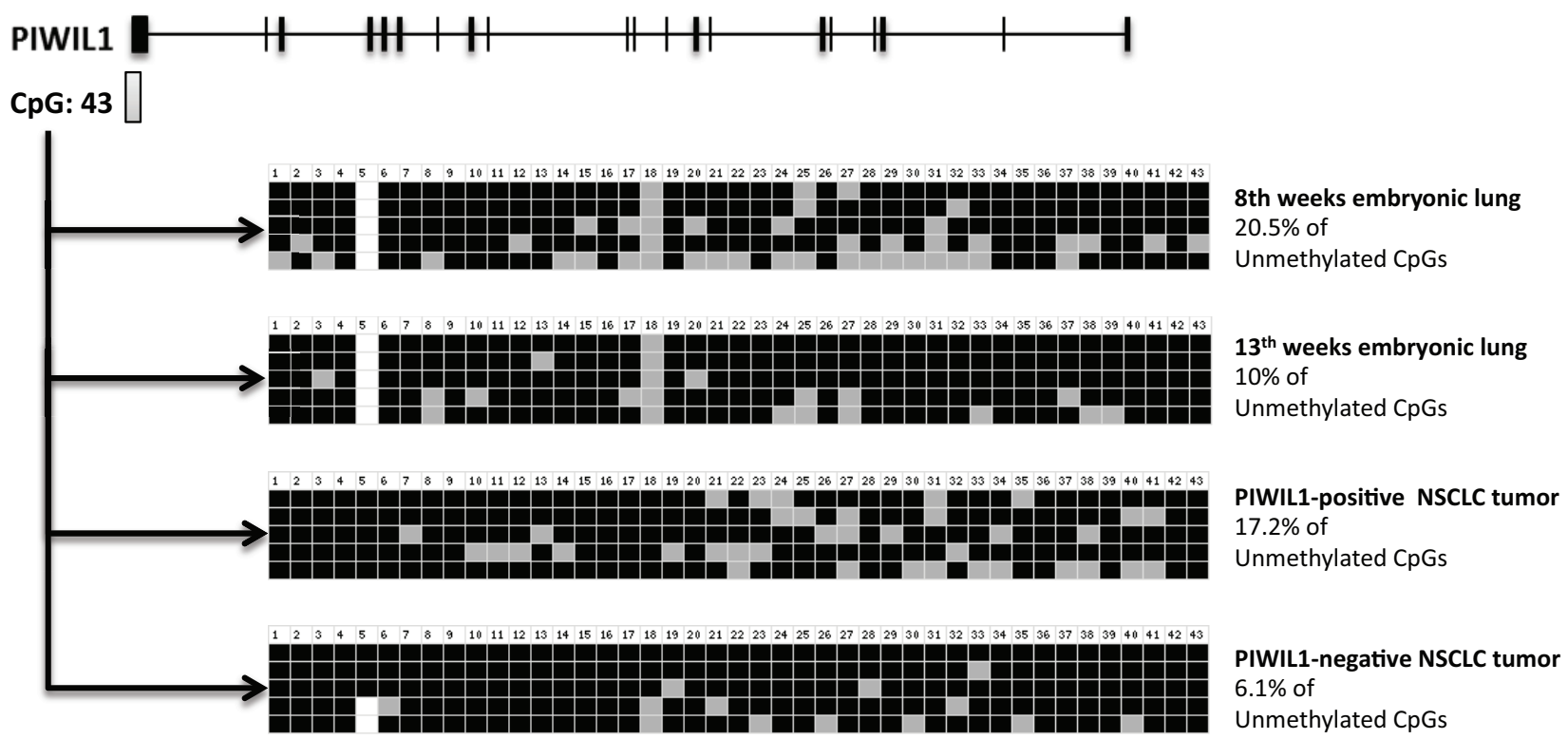

Figure 5: Study of the methylation of PIWI genes in lung cancer. (A) 5-Aza-dC treatment of H23 and A549 lung cancer cell lines showed an increase of the expression levels of the four PIWI genes. (B) Schematic representation of the PIWIL1 gene showing the presence of a $\mathrm{CpG}$ island close to the first exon of PIWIL1 gene. The bisulfite sequencing analysis of the $43 \mathrm{CpG}$ island showed differences between a PIWIL1-positive and a PIWIL1-negative case. 
changes in methylation levels in tumor samples. We observed a positive correlation of PIWIL4 levels with overall methylation $(r=0.267, p=0.044)$.

\section{Enriched gene signatures associated with PIWIL1 expression}

Since PIWIL1 was highly expressed during early lung development and PIWIL1 expression in tumor tissue was a marker of poor prognosis, we postulated that its expression could be associated with stem-cell characteristics. We therefore compared in silico the expression patterns associated with PIWIL1-positive patients vs PIWIL1negative patients. Using the public data available in GEO, we used the data from expression arrays performed in 58 NSCLC cases (GSE10245; PMID: 18486272). In these patients, we studied the expression of PIWIL1 gene and identified four patients expressing PIWIL1 (Figure 6A). We then compared gene expression between these four patients and the 54 PIWIL1-negative patients and were able to identify a 451 -gene signature (72 upregulated and 379 downregulated) associated with PIWIL1 expression (Supplementary Table 1). To study the functional relevance of this signature, we performed a Gene Set Enrichment Analysis (GSEA). GSEA identified several gene signatures (Supplementary Figure 1), including a gene signature associated with stem cells ("Wong_Adult_Tissue_Stem Module") (Figure 6B) [37]. Figure 6C displays all the genes included in the GSEA analysis after the filtering performed by the program; significant differences were observed between the PIWIL1-positive and PIWIL1negative group.

\section{DISCUSSION}

In the present study, we have examined whether the re-activation of the PIWI/piRNA pathway in tumor tissue influences patient outcome in NSCLC. The PIWI/ piRNA pathway was until recently thought to be active exclusively in germinal cells and in the first stages of embryonic development [14]. However, we have shown that PIWI expression can be detected during lung organogenesis, with a distinct expression pattern for each PIWI gene. Moreover, some PIWI genes can be detected in tumor and normal tissue - again with distinct expression patterns.

In piRNA biogenesis, we can distinguish two main pathways: the primary pathway and the secondary pathway. PIWIL1 participates exclusively in the primary pathway. The expression pattern of PIWIL1 indicates that while the primary pathway is very active during the early stages of lung embryogenesis, this pathway becomes downregulated during the differentiation of the embryonic and fetal lung and is finally inactivated in normal adult lung tissue. Interestingly, this pathway is reactivated in some patients, where it is a marker of poor prognosis. It has been shown in seminoma patients that this inactivation may be mediated by methylation [36]. To corroborate if this methylation-mediated inactivation would hold true in NSCLC, we treated two NSCLC cell lines with 5-Aza-dC, which led to the re-expression of all PIWI family members. Moreover, we analyzed a PIWIL1-positive and a PIWIL1negative tumor sample by bisulfite sequencing and found differences in the degree of methylation between the two samples. These results lead us to speculate that in lung cancer, PIWIL1 inactivation may well be mediated in part by methylation of its promoter region. Other mechanisms have also been shown to affect PIWIL1 expression. Reeves and coworkers found that PIWIL1 expression was higher in NSCLC cell lines (A549 and NCI-H1299) than in normal lung epithelial cells (CRL-9482) and that the expression of PIWIL1 could be activated by the oncogene RASSF1C [38].

In the present study, we have shown that the reactivation of PIWIL1 in tumors is associated with a stemcell expression signature. Liang et al. [39] have recently reported that PIWIL1 is essential to the maintenance of lung cancer stem cell populations. Knockdown of PIWIL1 compromised sphere formation ability in the stem cell population $\mathrm{SSC}^{\mathrm{lo}}$ Alde ${ }^{\mathrm{br}}$ and in vivo tumor growth in a nude mice model [39]. In a previous study, the same group showed that PIWIL1 gene silencing decreased proliferation and promoted apoptosis in lung cancer stem cells [40]. Although the prognostic significance of PIWIL1 expression has not previously been examined in NSCLC, it has been associated with poor outcome in other tumors, including glioma [26], pancreatic cancer [41], colorectal cancer [42], esophageal cancer [43], liver cancer [44], gastric cancer [45], and sarcomas [25, 46]. Furthermore, in glioma [47], breast cancer [48] and sarcomas [49], in vitro studies have shown that the suppression of PIWIL1 caused inhibition of cell growth by different mechanisms. Our finding that PIWIL1 expression was related to shorter TTR $(p=0.006)$ and OS $(p=0.0076)$ is along the same lines as these studies in other tumors and indicate that PIWIL1 re-expression could be a useful prognostic and therapeutic marker. Moreover, PIWIL1 protein levels can be easily detected in the pathology laboratory by immunohistochemistry.

PIWIL2 and PIWIL4 are active in the secondary pathway of piRNA biogenesis, where they participate in the "ping-pong" amplification cycle. PIWIL2 and PIWIL4 were both expressed in all samples - both in the embryonic and the adult normal and tumor tissue. In fact, during lung organogenesis (6-13 weeks), PIWIL2 and PIWIL4 expression is higher than that of PIWIL1 or PIWIL3. PIWIL4 expression remained high throughout the 6-13 weeks of development. In contrast, PIWIL2 expression increased significantly during the transition from the embryonic to the pseudoglandular stage of lung development, when there is a high rate of cell 
A

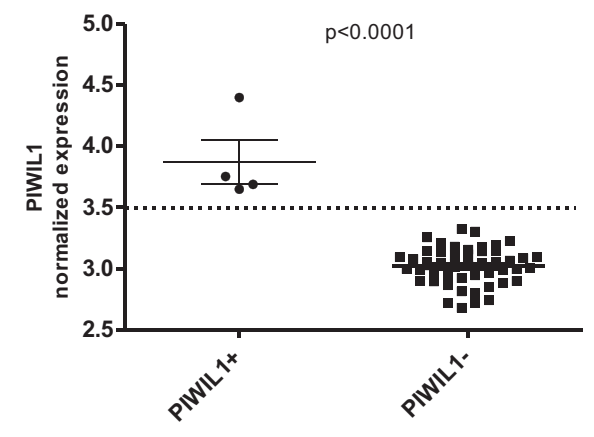

B

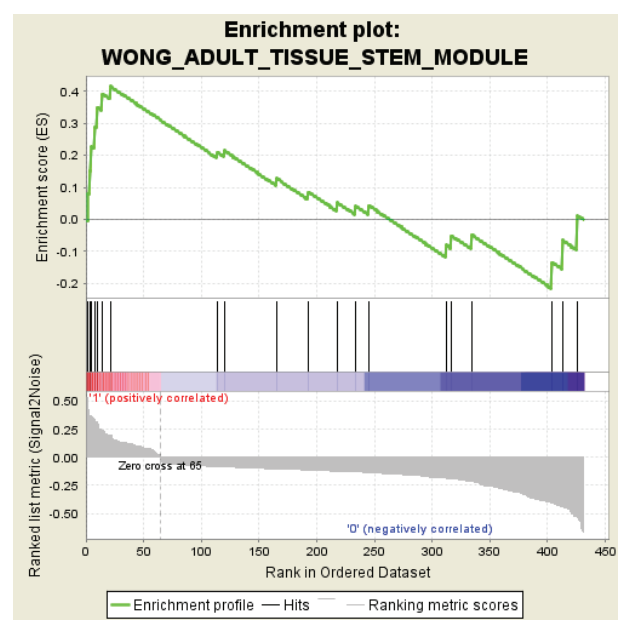

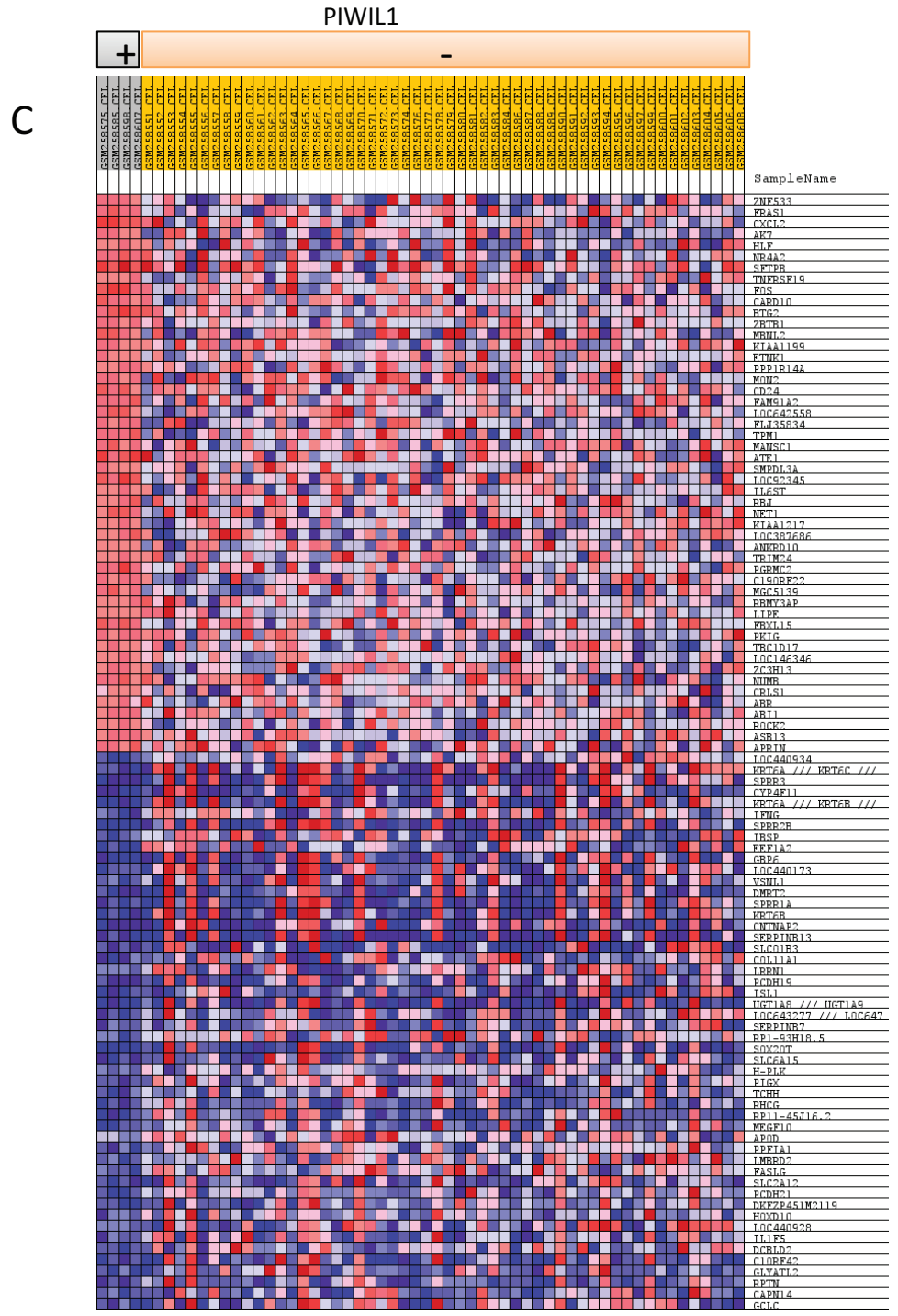

Figure 6: In silico analysis using GEO public data (GSE10245) to identify gene expression signatures associated with PIWIL1 expression. (A) Classification of patients according to PIWIL1 expression. Four patients were classified as PIWIL1-positive. (B) Enrichment plot showing that the gene expression signature associated with PIWIL1-positive patients is enriched in the Wong_Adult_ Tissue_Stem_Module. (C) Heat map of genes used by GSEA after filtering to perform the analysis. The heat map shows significant differences in the gene expression signature between PIWIL1-positive and PIWIL1-negative patients.

proliferation in order to produce the branching of the fetal lung. At embryonic weeks 6-8, endodermic buds appear, surrounded by mesenchymal mesodermic cells. At week 10, the endodermic cells acquire the characteristics of pseudostratified epithelium, and branching occurs. At week 12 , branching morphogenesis continues and the bronchial lumen can be observed, covered by a cubic endodermic epithelium [7]. The changes observed in PIWIL2 expression and the consistently high expression of PIWIL4 during this time could be related to this high rate of proliferation, highlighting the importance of the secondary piRNA pathway during this transition phase. Both PIWIL2 and PIWIL4 were also expressed in tumor and normal tissue, indicating that the secondary pathway is not inactivated in adult tissue. Interestingly, PIWIL2 and PIWIL4 were expressed at significantly lower levels in tumor than in paired normal tissue $(p<0.001)$. Importantly, patients with lower levels of PIWIL4 had shorter TTR $(p=0.048)$ and OS $(p=0.033)$.

It has been postulated that the secondary pathway is in charge of the maintenance of the pool of piRNAs of the cell, especially those targeting transposons [14]. This would match with the fact that PIWIL2 and PIWIL4 were detected in all our samples. Moreover, the downregulation of these genes in tumor samples could be related to an increase of transposon activities, resulting in genomic 
instability [50]. One of the mechanisms used by the piRNAs to silence transposons is DNA methylation [51]. It has been shown that PIWIL4 can modulate chromatin modifications through methylation of $\mathrm{H} 3 \mathrm{~K} 9$, specifically in the p16Ink4a locus [52]. In our study, we have observed a positive correlation between PIWIL4 levels and overall methylation. However, since the overall methylation levels were quantified using an ELISA method, it is not clear if the overall methylation corresponds to transposon regions or other transposable elements regulated by piRNAs. This interesting question warrants further study with more complex techniques.

In summary, we have examined the expression of PIWI genes in order to determine the activity and potential prognostic role of the PIWI/piRNA pathway in NSCLC. PIWIL1 participates in the primary pathway and PIWIL2 and PIWIL4 in the secondary pathway, both of which are active in NSCLC. The re-expression of the PIWIL1 gene, which can be confirmed by immunohistochemistry, is related to poor prognosis and is associated with a stemcell signature. The downregulation of PIWIL4 is also related to poor prognosis and is associated with lower methylation. Further investigation in a larger cohort of patients is warranted to validate these findings and to examine potential diagnostic and therapeutic approaches.

\section{MATERIALS AND METHODS}

\section{Study population}

Seventy-one fresh tumor samples and 71 paired normal tissue samples from NSCLC patients who underwent complete surgical resection in our institution were prospectively collected between June 2007 and March 2011. Immediately after surgery, samples were labelled, frozen at $-80^{\circ} \mathrm{C}$ and kept for further processing. Approval for the study was obtained from the Institutional Review Board of the Hospital Clinic of Barcelona, Spain. Written informed consent was obtained from each participant in accordance with the Declaration of Helsinki.

Twenty clinically aborted embryos and fetuses were donated with written informed consent to the Body Donation Service of the Human Anatomy and Embryology Department of the School of Medicine of University of Barcelona for morphological and molecular studies. The samples included lungs from the 6th to the 13th week of development. Lung samples were obtained under control of an Olympus stereo microscope SZ61. Samples for RNA extraction were frozen in liquid nitrogen and stored at $-80^{\circ} \mathrm{C}$ until analyzed.

\section{RNA extraction and mRNA quantification}

Total RNA was extracted from tumor and normal frozen tissue using Trizol (Life Technologies, Foster City, CA) according to the manufacturer's protocol. RNA from samples was quantified using a NanoDrop 1000 Spectrophotometer.

cDNA was synthesized from total RNA using the High Capacity cDNA Reverse Transcription Kit (Life Technologies) as per the manufacturer's protocol. TaqMan expression assays to determine RNA levels of PIWILI (Hs01041737_m1), PIWIL2 (Hs00216263_m1), PIWIL3 (Hs00908825_m1), and PIWIL4 (Hs00381509_m1) were supplied by Life Technologies. $\beta$-actin was used as housekeeping gene. RT-QPCR was performed in a total volume of $20 \mu \mathrm{l}$ in the ABI Prism 7500 Real Time PCR System (Life Technologies). All samples for each gene were run in triplicate for 40 cycles using the following master mix and thermal cycler conditions: $10 \mu \mathrm{l}$ of the TaqMan universal PCR master mix, $1 \mu$ of the primers and probes, $2 \mu \mathrm{l}$ of the cDNA and $7 \mu \mathrm{l}$ of the RNAse-free water; about $2 \min 50^{\circ} \mathrm{C}, 10 \min 95^{\circ} \mathrm{C}, 15 \mathrm{~s} 95^{\circ} \mathrm{C}$ and $1 \mathrm{~min} 60^{\circ} \mathrm{C}$. Fluorescent emission data were captured, and mRNA concentrations were quantified by using the critical threshold value and $2^{-\Delta \Delta \mathrm{Ct}}$ method.

\section{Immunohistochemistry}

Five- $\mu$ m-thick transverse sections of formalinfixed paraffin-embedded (FFPE) tissues were serially cut and mounted onto Dako Silanized Slides (Dako, Glostrup, Denmark). The immunohistochemical assay was performed as previously described [53] using rabbit polyclonal antibodies anti-human PIWIL1 (ab85125; Abcam, Cambridge, UK).

\section{Global methylation quantification}

Global methylation was determined as previously described $[54,55]$ in total DNA of all samples by a specific ELISA assay (MethylFlash ${ }^{\mathrm{TM}}$ Methylated DNA Quantification Kit, Epigentek, Farmingdale, NY), which is capable of recognizing the DNA methylated fraction. This test uses a monoclonal antibody against 5-methylcytosine (5-mC) to obtain the percentage of 5-mC in total DNA. The procedure was performed according to manufacturer's protocol.

\section{PIWIL1-associated gene expression signature}

Using arrays obtained from the Gene Expression Omnibus database (GSE10245), we examined the gene expression associated with patients who expressed PIWIL1. Raw files (.cel) were normalized using R statistical package (Institute for Statistics and Mathematics, Vienna, AU) and Bioconductor (www. bioconductor.org). The raw data was normalized using the rma method from the affy package (Bioconductor). After normalization, we obtained the expression data of PIWIL1 (probe: 214868_at). The samples that had higher expression than the mean and standard deviation of the population were classified as PIWIL1-positive. 
A $t$-test based on permutations was used to identify genes differentially expressed between patients with high and low PIWIL1 expression. The Gene Set Enrichment Analysis (GSEA v.2.1.0) program from "The Broad Institute" [56] was used to identify the enriched gene signatures associated with PIWIL1 expression.

\section{Cell culture and treatment}

Two NSCLC cell lines, H23 (American Type Culture Collection) and A549 (DSMZ - the German Resource Centre for Biological Material) were used. H23 and A549 cells were cultured in RPMI 1640 (Invitrogen) and DMEM (Invitrogen) respectively, containing 10\% fetal calf serum (Invitrogen).

For methylation analysis, both cell lines $\left(5 \times 10^{5}\right.$ cells) were plated $24 \mathrm{~h}$ prior to treatment. Cells were then treated daily with $1 \mu \mathrm{M} / 3 \mu \mathrm{M} / 10 \mu \mathrm{M}$ 5-Aza-2deoxycytidine (5-Aza-dC) during 6 days; on the 7 th day, the cells were harvested and gene expression was assessed.

\section{Bisulfite sequencing}

The methylation status of PIWIL1 was studied by bisulfite sequencing. Following sodium bisulfite treatment using Methylamp DNA Modification Kit (Epigentek, Farmingdale, NY), the $\mathrm{CpG}$ island located in the 5' region of PIWIL1 gene (UCSC GRCh37/hg19: chr12:130822361-130822696) was PCR amplified using Forward 5'-GGGGTTTTTTTGGTTT-3' and Reverse 5'-CACCTAACACCTCAACCTAACC-3' primers. PCR products were subcloned using the TOPO TA Cloning kit (Invitrogen, Life Technologies) into pCR2.1-Topo TA Vector plasmid and 5 candidate plasmid clones were sequenced. The resulting sequences were analyzed using BISMA [57].

\section{Statistical analyses}

The primary endpoints of the study were to assess time to relapse (TTR) and overall survival (OS), calculated from the time of surgical treatment to the date of relapse or death from any cause, respectively. TTR and OS were calculated using the Kaplan-Meier method and compared using the log-rank test. Optimal cut-off points of gene expression data for TTR were assessed by means of maximally selected log-rank statistics [24] using the Maxstat package (version 2.8.1; $\mathrm{R}$ statistical package) and confirmed by the KaplanMeier test. All variables with a $p$-value $\leq 0.1$ in the univariate analysis were included in a Cox multivariate analysis in order to calculate the independent odds ratios (ORs) for TTR and OS. All statistical analyses were performed with PASW Statistics 18 (SPSS Inc., Chicago, IL, USA) and $\mathrm{R}$ version 2.13. Statistical significance was set at $p \leq 0.05$.

\section{ACKNOWLEDGMENTS}

We thank Dr. S. Barambio, Y. Trejo and R. Lopez for their support in embryologic aspects. This work was supported by grants from AECC-Catalunya 2013 (sponsored by Mat Holding), SDCSD of University of Barcelona, FIS 12/405, SEPAR y SOCAP. Anna Cordeiro is an APIF fellow of the University of Barcelona and Oriol Caritg is an AECC fellow.

\section{REFERENCES}

1. Siegel R, Naishadham D, Jemal A. Cancer statistics, 2013. CA: a cancer journal for clinicians. 2013; 63:11-30

2. Molina JR, Yang P, Cassivi SD, Schild SE, Adjei AA. Nonsmall cell lung cancer: epidemiology, risk factors, treatment, and survivorship. Mayo Clinic Proceedings: Elsevier. 2008; pp. 584-594.

3. Reya T, Morrison SJ, Clarke MF, Weissman IL. Stem cells, cancer, and cancer stem cells. Nature. 2001; 414:105-111.

4. Monzo M, Navarro A, Bandres E, Artells R, Moreno I, Gel B, Ibeas R, Moreno J, Martinez F, Diaz T. Overlapping expression of microRNAs in human embryonic colon and colorectal cancer. Cell research. 2008; 18:823-833.

5. Hu M, Shivdasani RA. Overlapping gene expression in fetal mouse intestine development and human colorectal cancer. Cancer research. 2005; 65:8715-8722.

6. Borczuk AC, Gorenstein L, Walter KL, Assaad AA, Wang L, Powell CA. Non-small-cell lung cancer molecular signatures recapitulate lung developmental pathways. The American journal of pathology. 2003; 163:1949-1960.

7. Navarro A, Marrades RM, Viñolas N, Quera A, Agustí C, Huerta A, Ramirez J, Torres A, Monzo M. MicroRNAs expressed during lung cancer development are expressed in human pseudoglandular lung embryogenesis. Oncology. 2009; 76:162-169.

8. Powers S, Mu D. Genetic similarities between organogenesis and tumorigenesis of the lung. CELL CYCLE-LANDES BIOSCIENCE-. 2008; 7:200.

9. Esteller M. Non-coding RNAs in human disease. Nature Reviews Genetics. 2011; 12:861-874.

10. Ghildiyal M, Zamore PD. Small silencing RNAs: an expanding universe. Nature Reviews Genetics. 2009; 10:94-108.

11. Sasaki T, Shiohama A, Minoshima S, Shimizu N. Identification of eight members of the Argonaute family in the human genome区. Genomics. 2003; 82:323-330.

12. Girard A, Sachidanandam R, Hannon GJ, Carmell MA. A germline-specific class of small RNAs binds mammalian Piwi proteins. Nature. 2006; 442:199-202.

13. Mei Y, Clark D, Mao L. Novel dimensions of piRNAs in cancer. Cancer letters. 2013; 336:46-52. 
14. Luteijn MJ, Ketting RF. PIWI-interacting RNAs: from generation to transgenerational epigenetics. Nature Reviews Genetics. 2013; 14:523-534.

15. Gerstl MP, Hackl M, Graf AB, Borth N, Grillari J. Prediction of transcribed PIWI-interacting RNAs from CHO RNAseq data. Journal of biotechnology. 2013; 166:51-57.

16. Cheng J, Guo J-M, Xiao B-X, Miao Y, Jiang Z, Zhou H, Li Q-N. piRNA, the new non-coding RNA, is aberrantly expressed in human cancer cells. Clinica Chimica Acta. 2011; 412:1621-1625.

17. Cheng J, Deng H, Xiao B, Zhou H, Zhou F, Shen Z, Guo J. piR-823, a novel non-coding small RNA, demonstrates in vitro and in vivo tumor suppressive activity in human gastric cancer cells. Cancer letters. 2012; 315:12-17.

18. Siomi MC, Sato K, Pezic D, Aravin AA. PIWI-interacting small RNAs: the vanguard of genome defence. Nature reviews Molecular cell biology. 2011; 12:246-258.

19. Robine N, Lau NC, Balla S, Jin Z, Okamura K, KuramochiMiyagawa S, Blower MD, Lai EC. A broadly conserved pathway generates 3' UTR-directed primary piRNAs. Current Biology. 2009; 19:2066-2076.

20. Siddiqi S, Matushansky I. Piwis and piwi-interacting RNAs in the epigenetics of cancer. Journal of cellular biochemistry. 2012; 113:373-380.

21. Vagin VV, Sigova A, Li C, Seitz H, Gvozdev V, Zamore PD. A distinct small RNA pathway silences selfish genetic elements in the germline. Science. 2006; 313:320-324.

22. Qiao D, Zeeman A-M, Deng W, Looijenga L, Lin H. Molecular characterization of hiwi, a human member of the piwi gene family whose overexpression is correlated to seminomas. Oncogene. 2002; 21:3988-3999.

23. Aravin AA, Hannon GJ, Brennecke J. The Piwi-piRNA pathway provides an adaptive defense in the transposon arms race. Science. 2007; 318:761-764.

24. Houwing S, Kamminga LM, Berezikov E, Cronembold D, Girard A, Van Den Elst H, Filippov DV, Blaser H, Raz E, Moens CB. A role for Piwi and piRNAs in germ cell maintenance and transposon silencing in Zebrafish. Cell. 2007; 129:69-82.

25. Taubert H, Greither T, Kaushal D, Würl P, Bache M, Bartel F, Kehlen A, Lautenschläger C, Harris L, Kraemer K. Expression of the stem cell self-renewal gene Hiwi and risk of tumour-related death in patients with soft-tissue sarcoma. Oncogene. 2006; 26:1098-1100.

26. Sun G, Wang Y, Sun L, Luo H, Liu N, Fu Z, You Y. Clinical significance of Hiwi gene expression in gliomas. Brain research. 2011; 1373:183-188.

27. Liu X, Sun Y, Guo J, Ma H, Li J, Dong B, Jin G, Zhang J, $\mathrm{Wu}$ J, Meng L. Expression of hiwi gene in human gastric cancer was associated with proliferation of cancer cells. International journal of cancer. 2006; 118:1922-1929.

28. Lee JH, Schütte D, Wulf G, Füzesi L, Radzun H-J, Schweyer S, Engel W, Nayernia K. Stem-cell protein
Piwil2 is widely expressed in tumors and inhibits apoptosis through activation of Stat3/Bcl-XL pathway. Human molecular genetics. 2006; 15:201-211.

29. Liu JJ, Shen R, Chen L, Ye Y, He G, Hua K, Jarjoura D, Nakano T, Ramesh GK, Shapiro CL. Piwil2 is expressed in various stages of breast cancers and has the potential to be used as a novel biomarker. International journal of clinical and experimental pathology. 2010; 3:328.

30. Unhavaithaya Y, Hao Y, Beyret E, Yin H, KuramochiMiyagawa S, Nakano T, Lin H. MILI, a PIWI-interacting RNA-binding protein, is required for germ line stem cell self-renewal and appears to positively regulate translation. Journal of Biological Chemistry. 2009; 284:6507-6519.

31. Cox DN, Chao A, Baker J, Chang L, Qiao D, Lin H. A novel class of evolutionarily conserved genes defined by piwi are essential for stem cell self-renewal. Genes \& development. 1998; 12:3715-3727.

32. Siddiqi S, Terry M, Matushansky I. Hiwi mediated tumorigenesis is associated with DNA hypermethylation. PloS one. 2012; 7:e33711.

33. Zeng Y, Qu L-k, Meng L, Liu C-y, Dong B, Xing X-f, $\mathrm{Wu}$ J, Shou C-c. HIWI expression profile in cancer cells and its prognostic value for patients with colorectal cancer. Chinese Medical Journal-Beijing. 2011; 124:2144.

34. Suzuki R, Honda S, Kirino Y. PIWI expression and function in cancer. Frontiers in genetics. 2012; 3:204.

35. Hothorn T, Lausen B. On the exact distribution of maximally selected rank statistics. Computational Statistics \& Data Analysis. 2003; 43:121-137.

36. Ferreira HJ, Heyn H, del Muro XG, Vidal A, Larriba S, Muñoz C, Villanueva A, Esteller M. Epigenetic loss of the PIWI/piRNA machinery in human testicular tumorigenesis. Epigenetics. 2014; 9:113.

37. Wong DJ, Liu H, Ridky TW, Cassarino D, Segal E, Chang HY. Module map of stem cell genes guides creation of epithelial cancer stem cells. Cell stem cell. 2008; 2:333-344.

38. Reeves ME, Baldwin ML, Aragon R, Baldwin S, Chen S-T, Li X, Mohan S, Amaar YG. RASSF1C modulates the expression of a stem cell renewal gene, PIWIL1. BMC research notes. 2012; 5:239.

39. Liang D, Dong M, Hu L-J, Fang Z-H, Xu X, Shi E-H, Yang Y-J. Hiwi knockdown inhibits the growth of lung cancer in nude mice. Asian Pacific Journal of Cancer Prevention. 2013; 14:1067-1072.

40. Liang D, Fang Z, Dong M, Liang C, Xing C, Zhao J, Yang Y. Effect of RNA interference-related HiWi gene expression on the proliferation and apoptosis of lung cancer stem cells. Oncology letters. 2012; 4:146-150.

41. Grochola L, Greither $T$, Taubert $H$, Möller $P$, Knippschild U, Udelnow A, Henne-Bruns D, Würl P. The stem cell-associated Hiwi gene in human adenocarcinoma 
of the pancreas: expression and risk of tumour-related death. British journal of cancer. 2008; 99:1083-1088.

42. Liu C, Qu L, Dong B, Xing X, Ren T, Zeng Y, Jiang B, Meng L, Wu J, Shou C. Combined phenotype of 4 markers improves prognostic value of patients with colon cancer. The American journal of the medical sciences. 2012; 343:295-302.

43. He W, Wang Z, Wang Q, Fan Q, Shou C, Wang J, Giercksky K-E, Nesland JM, Suo Z. Expression of HIWI in human esophageal squamous cell carcinoma is significantly associated with poorer prognosis. BMC cancer. 2009; 9:426.

44. Zhao YM, Zhou JM, Wang LR, He HW, Wang XL, Tao ZH, Sun HC, Wu WZ, Fan J, Tang ZY. HIWI is associated with prognosis in patients with hepatocellular carcinoma after curative resection. Cancer. 2012; 118:2708-2717.

45. Wang Y, Liu Y, Shen X, Zhang X, Chen X, Yang C, Gao H. The PIWI protein acts as a predictive marker for human gastric cancer. International journal of clinical and experimental pathology. 2012; 5:315.

46. Taubert H, Würl P, Greither T, Kappler M, Bache M, Bartel F, Kehlen A, Lautenschläger C, Harris L, Kaushal D. Stem cell-associated genes are extremely poor prognostic factors for soft-tissue sarcoma patients. Oncogene. 2007; 26:7170-7174.

47. Wang X, Tong X, Gao H, Yan X, Xu X, Sun S, Wang Q, Wang J. Silencing HIWI suppresses the growth, invasion and migration of glioma cells. International journal of oncology. 2014; 45:2385-2392.

48. Wang D, Wang Z, Wang L, Song Y, Zhang G. Overexpression of hiwi promotes growth of human breast cancer cells. Asian Pacific journal of cancer prevention: APJCP. 2013; 15:7553-7558.

49. Siddiqi S. The Role of Hiwi in Stem Cell Maintenance and Sarcomagenesis. Columbia University Academic Commons. http://hdl.handle.net/10022/AC:P:13571. 2012; 1:1-134.
50. Peng JC, Lin H. Beyond transposons: the epigenetic and somatic functions of the Piwi-piRNA mechanism. Current opinion in cell biology. 2013; 25:190-194.

51. Aravin AA, Bourc'his D. Small RNA guides for de novo DNA methylation in mammalian germ cells. Genes \& development. 2008; 22:970-975.

52. Sugimoto K, Kage H, Aki N, Sano A, Kitagawa H, Nagase T, Yatomi Y, Ohishi N, Takai D. The induction of H3K9 methylation by PIWIL4 at the p16 $6^{\text {Ink4a }}$ locus. Biochemical and biophysical research communications. 2007; 359:497-502.

53. Artells R, Navarro A, Diaz T, Monzó M. Ultrastructural and Immunohistochemical Analysis of Intestinal Myofibroblasts During the Early Organogenesis of the Human Small Intestine. The Anatomical Record. 2011; 294:462-471.

54. Calvo X, Nomdedeu M, Navarro A, Tejero R, Costa D, Muñoz C, Pereira A, Peña O, Risueño RM, Monzó M. High levels of global DNA methylation are an independent adverse prognostic factor in a series of 90 patients with de novo myelodysplastic syndrome. Leukemia Research. 2014; 38:874-881.

55. Fernández de Larrea C, Martín-Antonio B, Cibeira MT, Navarro A, Tovar N, Díaz T, Rosiñol L, Monzó M, UrbanoIspizua A, Bladé J. Impact of global and gene-specific DNA methylation pattern in relapsed multiple myeloma patients treated with bortezomib. Leukemia Research. 2013; 37:641-646.

56. Subramanian A, Tamayo P, Mootha VK, Mukherjee S, Ebert BL, Gillette MA, Paulovich A, Pomeroy SL, Golub TR, Lander ES. Gene set enrichment analysis: a knowledgebased approach for interpreting genome-wide expression profiles. Proceedings of the National Academy of Sciences of the United States of America. 2005; 102:15545-15550.

57. Rohde C, Zhang Y, Reinhardt R, Jeltsch A. BISMA-Fast and accurate bisulfite sequencing data analysis of individual clones from unique and repetitive sequences. BMC bioinformatics. 2010; 11:230. 